\title{
La tecnología cerámica de los niveles IV y III en el yacimiento de Kobaederra (Cortézubi, Bizkaia). Aprovisionamiento y modificación de las materias primas
}

\author{
Pottery technology of levels IV and III in Kobaederra site (Cortézubi, Bizkaia). Supply and \\ modification of raw materials
}

\author{
Miriam Cubas (*) \\ Manuel García-Heras (**) \\ David Méndez $(* * *)$ \\ Imanol de Pedro $(* * * *)$
}

\section{RESUMEN}

El estudio tecnológico del material cerámico requiere de la aplicación de técnicas de análisis arqueométrico que permitan un acercamiento a su mineralogía y composición química, con la finalidad de identificar las etapas de su secuencia de elaboración. Este artículo presenta el análisis tecnológico de la cerámica neolítica documentada en los niveles inferiores del yacimiento de Kobaederra (Cortézubi, Bizkaia), a partir de su análisis mineralógico (petrografía y difracción de rayos $\mathrm{X}, \mathrm{DRX}$ ) y geoquímico (microscopio electrónico de barrido-espectómetro de dispersión de energía, MEB-EDS). Su objetivo es discriminar las potenciales áreas de aprovisionamiento de las materias primas y su modificación mediante la adición de desgrasantes. Por último, se discuten las implicaciones de los resultados obtenidos en relación con el resto de las evidencias arqueológicas disponibles para los niveles IV y III de Kobaederra.

\begin{abstract}
The technological study of ceramic materials requires the application of archaeometric analytical techniques to
\end{abstract}

(*) Sociedad de Ciencias Aranzadi. C/ Zorroagagaina 11. 20014 Donostia-San Sebastián.

Correo e.: miriam.cubas@hotmail.com

(**) GI Arqueometría y Conservación de Vidrios y Materiales Cerámicos. Instituto de Historia. CCHS-CSIC. C/ Albasanz 26-28. 28037 Madrid. Correo e.: manuel.gheras@cchs.csic.es

(***) Laboratorio de la División de Ciencia e Ingeniería de los Materiales, Universidad de Cantabria, E. T. S. Ingenieros de Caminos. Avda. de los Castros s/n. 39005 Santander.

Correo e.: mendezd@unican.es

(****) Dpto. Ciencias de la Tierra y Física de la Materia Condensada (CITIMAC)-Universidad de Cantabria. Facultad de Ciencias. Avda. de los Castros s/n. 39005 Santander.

Correo e.: manuel.depedro@unican.es

\author{
Lydia Zapata (*****) \\ Juan José Ibáñez (******) \\ Jesús Emilio González Urquijo (*******)
}

approach both their mineralogy and chemical composition, with the aim of identifying steps in their production sequence. This paper presents the technological analysis of the Neolithic pottery documented in the lower levels of the Kobaederra site (Cortézubi, Bizkaia) on the basis of their mineralogical (petrography and X-ray diffraction, $X R D)$ and geochemical (SEM-EDS) analysis. Its goal is to discriminate the possible areas of raw materials supply and their modification through the addition of tempers. Finally, the implications of the results in relation to the rest of available archaeological evidences from the $I V$ and the III levels of the Kobaederra site are discussed.

Palabras clave: Cerámica; Neolítico; Península Ibérica; Arqueometría; Secuencia de manufactura; Tecnología; Desgrasante; Chamota; Calcita; Sílex.

Key words: Pottery; Neolithic; Iberian Peninsula; Archaeometry; Manufacturing process; Technology; Temper; Grog; Calcite; Flint.

\section{INTRODUCCIÓN}

El estudio sobre el Neolítico en la región cantábrica se ha intensificado de forma considerable

(*****) Universidad del País Vasco (UPV-EHU), Facultad de Letras. C/ F. Tomás y Valiente s/n. 01066 Vitoria-Gasteiz. Correo e.: lydia.zapata@ehu.es

$(* * * * *)$ Institución Milá i Fontanals, CSIC. C/ Egipcíaques 15. 08001 Barcelona. Correo e.: ibanezjj@imf.csic.es

(*******) Instituto Internacional de Investigaciones Prehistóricas de Cantabria (IIIPC)-Universidad de Cantabria. Edif. Interfacultativo. Avda. de los Castros s/n. 39005 Santander. Correo e.: jesuse.gonzalez@unican.es

Recibido: 5-IV-2011; aceptado: 31-V-2011. 
en las últimas décadas del siglo XX. Los distintos proyectos de investigación se han centrado en precisar y establecer la aparición de las especies domésticas en la región (Arias y Altuna 1999; Zapata 2002; Peña-Chocarro et al. 2005; Altuna y Mariezkurrena 2009), en la cronología del proceso (Arias et al. 2000), en los materiales cerámicos (Alday 2003; Cubas 2008) y en el propio proceso de neolitización (Arias 2007; Arias et al. 2000). Ello se ha visto favorecido por la excavación de nuevos depósitos como Los Canes(1), Los Gitanos (Ontañón 2005), El Mirón (González Morales y Straus 2000), Kobaederra (Zapata et al. 1997) y Herriko Barra (Iriarte et al. 2005).

Gracias a esos avances, se han adscrito a la primera mitad del $\mathrm{V}$ milenio cal AC las evidencias cerámicas más antiguas de la región cantábrica. Proceden de los yacimientos de Los Canes, E1 Mirón (González Morales y Straus 2000), Arenillas (Bohigas y Muñoz 2002), Los Gitanos (Ontañón 2005), Arenaza (Apellániz y Altuna 1975) y Kobaederra (Zapata et al. 1997). El análisis de parte de estas cerámicas se ha integrado en las síntesis sobre el Neolítico en la región (Arias 1991) o en ensayos de sistematización de sus caracteres morfoestilísticos (Alday 2003). Algunas cerámicas han sido objeto de un estudio monográfico como las halladas en El Mirón(2) o Los Gitanos (Cubas y Ontañón 2009).

El elevado índice de fragmentación y la escasa representatividad morfológica de los conjuntos cerámicos han impedido su sistematización atendiendo a criterios morfoestilísticos (Cubas 2008). En general muestran un buen estado de conservación con leves concreciones calcáreas en sus superficies o coloraciones debidas a infiltraciones, ya que proceden de entornos kársticos.

En este artículo se considera la cerámica como manufactura, inserta en una secuencia tecnológica de elaboración que transforma la arcilla en un recipiente con unas características físico-químicas concretas. En función de este enfoque los objetivos fueron el reconocimiento de las potenciales áreas de aprovisionamiento de las materias

(1) Arias, P. 2002: La Cueva de Los Canes (Asturias). Los últimos cazadores de la Península Ibérica ante la muerte. Memoria de Cátedra. Dpto. de Ciencias Históricas. Universidad de Cantabria. Santander. Inédito.

(2) Vega Maeso, C. 2006: La cerámica de la cueva de El Mirón. Manufactura, morfología y abandono. Trabajo de investigación de Tercer Ciclo. Universidad de Cantabria. Santander. Inédito. primas empleadas en la elaboración de las cerámicas de los niveles inferiores del yacimiento de Kobaederra y la discriminación de sus secuencias de manufactura.

\section{EL DEPÓSITO ARQUEOLÓGICO DE KOBAEDERRA (CORTÉZUBI, BIZKAIA)}

Kobaederra (coordenadas UTM-30T WGS84x: 530927; y: 4798992 y 260 m.s.n.m) se sitúa en el municipio de Cortézubi (Bizkaia) (Fig. 1). Es una cavidad de gran tamaño, unos $25 \mathrm{~m}$ de ancho por $6 \mathrm{~m}$ de alto en su parte central, abierta en las calizas paraarrecifales del Aptiense (Cretácico Inferior) del monte Aritzgane. Se ubica en la ría de Guernica que se dispone con una dirección N-S a favor de los materiales blandos y erosionados del Keuper (Fig. 1). El núcleo de la ría está constituido por materiales triásicos -en su mayoría arcillas abigarradas, rocas de textura ofítica y yesos- y en los flancos se disponen depósitos jurásicos y cretácicos en una serie casi continua (IGME 1973a, 1973b). El Jurásico, en la parte oriental de la cuenca vasco-cantábrica, es predominantemente marino y carbonatado, con eventuales unidades detríticas (Garmendia y Robles 1991: 9). Este carácter carbonatado caracteriza a las calizas arrecifales y paraarrecifales (Cretácico Inferior) tan abundantes en el entorno del yacimiento con alternancia de calizas, margas y argilolitas calcáreas masivas (IGME 1973a, 1973b). Por último, los depósitos cuaternarios están ampliamente documentados en la ría y en su mayoría son materiales holocenos depositados en el estuario que se formó como consecuencia de la transgresión marina (EVE 2003).

Los materiales aquí estudiados proceden de las intervenciones arqueológicas desarrolladas entre 1995 y 1999 (Zapata et al. 1997; Zapata et al. 2000). La excavación se organizó en unidades de $1 \mathrm{~m}^{2}$, subdivididas en cuadrantes, y se realizó mediante tallas de espesor variable (entre 3 y 6 $\mathrm{cm}$ ) siguiendo el buzamiento reconocido en el sedimento. Los materiales registrados fueron industria lítica, ósea, cerámica, restos faunísticos determinables o mayores de $4 \mathrm{~cm}$ y muestras antracológicas, carpológicas y malacológicas. Se recogieron en bolsas individuales indicando su posición topográfica. Todo el sedimento se flotó 


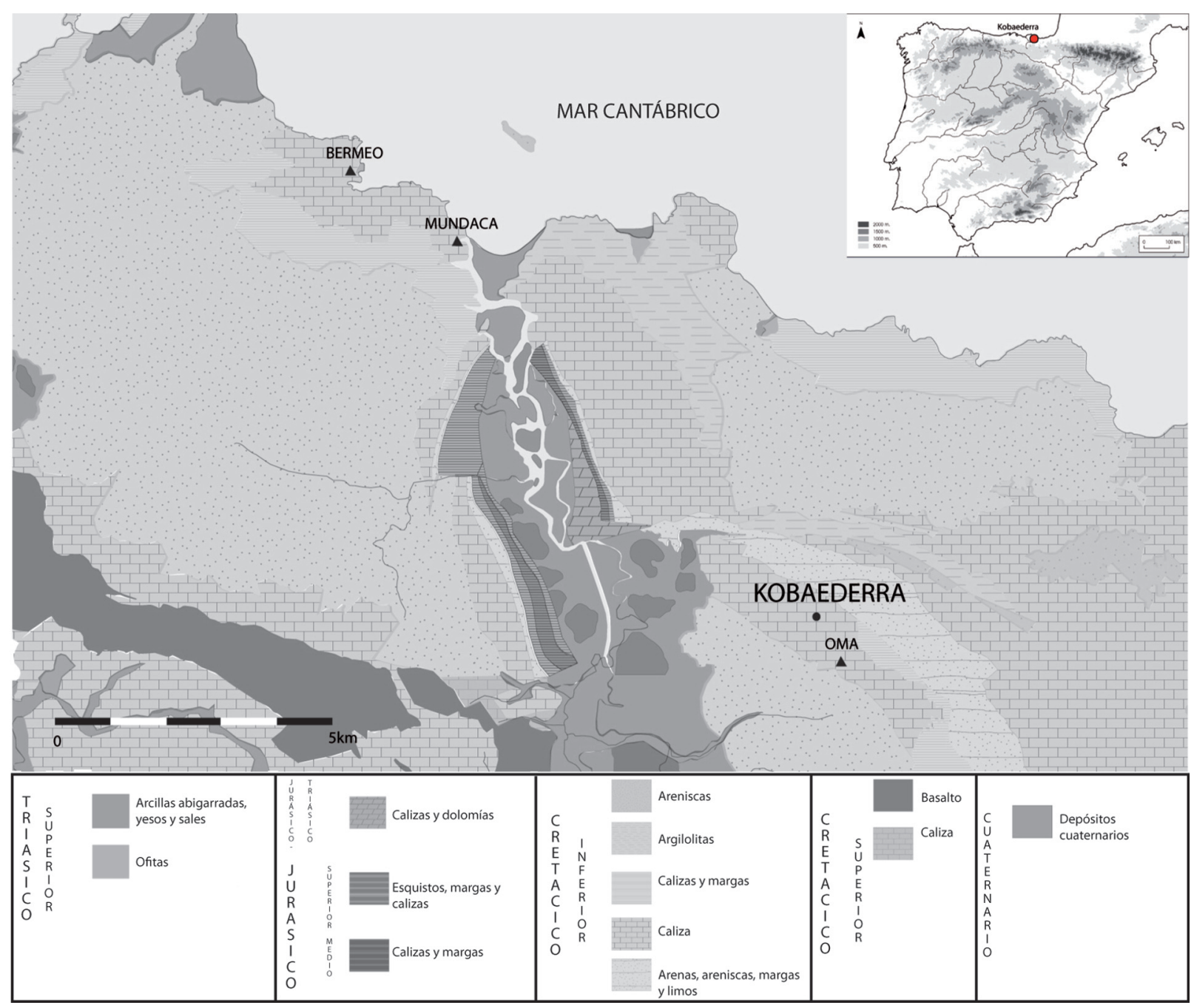

Fig. 1. Localización del yacimiento de Kobaederra y principales rasgos geológicos del entorno. Mapa realizado a partir de las hojas 37, 38 y 62 del Mapa Geológico de España, Instituto Geológico y Minero, serie MAGNA 50, escala $1: 50.000$

para recuperar los macrorrestos botánicos, seleccionándose el residuo insoluble.

La parte superficial de la secuencia se definió como nivel I (Fig. 2) con una potencia variable entre 34 y $62 \mathrm{~cm}$ de espesor. El sedimento es de fracción limo-arcillosa, con capas costrificadas y de escasa compacidad. Este nivel se superpone a una capa estalagmítica que alcanza los $20 \mathrm{~cm}$ de espesor en algunas zonas. La datación absoluta (OxA-6960) (Tab. 1) sitúa su formación entre el 3330 y el 2910 cal AC.

El nivel II, infrayacente y separado del anterior por una costra estalagmítica, tiene características sedimentológicas y arqueológicas simi- lares. Se extiende por la práctica totalidad del área excavada con una potencia entre 18 y 54 $\mathrm{cm}$ de espesor y una costra estalagmítica a base. Según las dataciones absolutas disponibles se formó en un momento avanzado del $\mathrm{V}$ milenio cal AC (Beta-126686 y UBAR-472) (Tab. 1). Un enterramiento en fosa cortaba al nivel II y una pequeña costra lo separaba del infrayacente nivel III. La datación directa de los restos humanos (OxA-29109: 2040-1700 cal AC) (Tab. 1) sugiere que esta fosa intrusiva puede haber afectado la integridad del depósito del nivel II y provocado la remoción de los materiales de ambos niveles. 

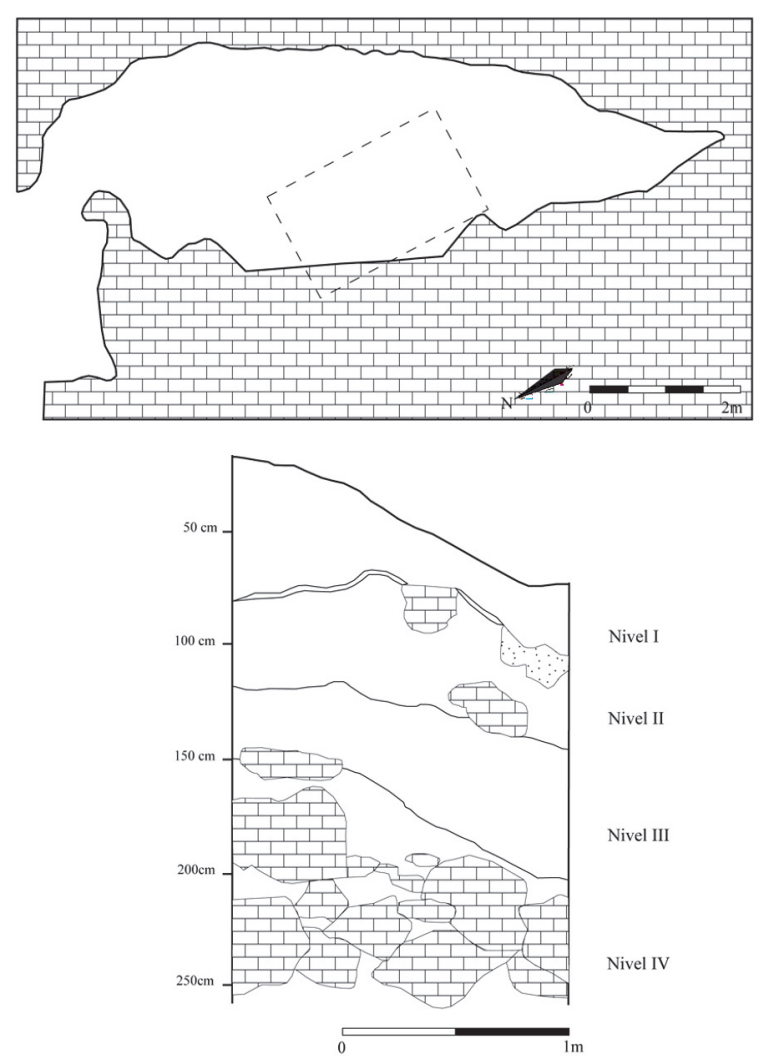

Fig. 2. Yacimiento de Kobaederra (Cortézubi, Bizkaia). Planta y perfil oriental del sondeo de la galería suroeste.

El nivel III es de fracción arcillosa, muy compacto, con cenizas y carbones que le confieren una tonalidad marrón-grisácea. Tiene una potencia entre 35 y $55 \mathrm{~cm}$ de espesor, con una disposición más horizontal que los dos anteriores. Se formó en el período comprendido entre el 5310 y 4230 cal AC (UBAR-471) (Tab. 1). El nivel IV, la parte inferior de la secuencia, es un sedimento de fracción arcillosa-limosa y de unos $20 \mathrm{~cm}$ de espesor. Según las dataciones absolutas disponibles (UBAR-470 y AA-29110) (Tab. 1) se formó a lo largo del $\mathrm{V}$ milenio cal AC.

Las dataciones absolutas no son muy concluyentes (Tab. 1). Se han seleccionado los conjuntos cerámicos de los niveles inferiores de la secuencia porque constituyen las cerámicas más antiguas documentadas en el yacimiento, en torno a mediados del $\mathrm{V}$ milenio cal $\mathrm{AC}$, con cronologías similares en el resto de la región. A pesar de la aparente homogeneidad observada en las dataciones absolutas del nivel II, se ha decidido excluir su conjunto cerámico ya que la fosa posterior pudo haber afectado a la integridad del depósito.

El conjunto arqueológico de los niveles IV y III del yacimiento evidencia una escasez de restos líticos y cerámicos en comparación con la gran abundancia de fauna de mamíferos. Está representada por animales domésticos, básicamente ovinos, $\mathrm{y}$, en menor proporción salvajes, especialmente Cervus (Altuna y Mariezkurrena 2009). Los restos malacológicos son muy numerosos: unos 6.000 por $\mathrm{m}^{2}$ con un dominio de los bivalvos, Ostrea, $R u$ ditapes y Scrobicularia (Gutiérrez Zugasti 2009). El análisis de macrorrestos vegetales constata trigo vestido (Triticum dicoccum), cebada, leguminosas, avellanas y bellotas, así como el uso mayoritario de madera de Quercus subg. Quercus (grupo de robles caducifolios y marcescentes) como combustible (Zapata 2002: 94).

\begin{tabular}{|c|c|c|c|c|c|c|}
\hline Nivel & Muestra & Laboratorio & Datación BP & Desv. Típica & $\begin{array}{c}\text { Calibración } \\
\text { cal AC/2 }\end{array}$ & Bibliografía \\
\hline I & Cereal & OxA-6960 & 4405 & 55 & $3330-2910$ & Arias et al. 1999 \\
II & Hueso humano & AA-29109 & 3545 & 60 & $2040-1700$ & Arias et al. 1999 \\
II & Carbón & UBAR-472 & 5200 & 110 & $4320-3770$ & Zapata et al. 1997 \\
II & Carbón & Beta-126686 & 5460 & 60 & $4450-4080$ & Zapata 2002 \\
III & Carbón & UBAR-471 & 5820 & 240 & $5310-4230$ & Zapata et al. 1997 \\
IV & Cereal & AA-29110 & 5375 & 90 & $4360-3990$ & Zapata 2002 \\
IV & Carbón & UBAR-470 & 5630 & 100 & $4710-4270$ & Zapata et al. 1997 \\
\hline
\end{tabular}

Tab. 1. Dataciones absolutas disponibles para la secuencia estratigráfica (yacimiento de Kobaederra, Cortézubi, Bizkaia). Las calibraciones corresponden a la curva IntCal09 para muestras terrestres (Reimer et al. 2009). Se ha utilizado el programa OxCa14.1.5 (Ramsey 2001, 2009) y se muestran en años cal AC con un intervalo de probabilidad $2 \sigma$. 


\section{METODOLOGÍA}

La metodología se basó en tres niveles de análisis: macroscópico, mineralógico y composicional. Debido a la gran fragmentación del material, el fragmento cerámico fue la unidad de análisis.

El análisis macroscópico se llevó a cabo sin tratamiento previo y se realizó en todos los fragmentos cerámicos recuperados en la excavación $(\mathrm{n}=417$, Tab. 2), fueran partes morfológicas representativas o indeterminados. Se observaron las variables: cuerpo cerámico y matriz arcillosa, inclusiones, tratamiento de superficies, decoración y las alteraciones postdeposicionales. Como resultado del análisis macroscópico se establecieron grupos de referencia, que después se tomaron como base para el análisis mineralógico mediante lámina delgada. Algunos autores critican este sistema de muestreo (Cowgill 1964) ya que los grupos de referencia se establecen mediante criterios subjetivos. Sin embargo, este tipo de muestreo permite reconocer toda la variabilidad del conjunto cerámico estudiado y asegura que, al menos, una muestra de cada grupo esté finalmente representada (Neff 1993: 30). Un procedimiento similar se ha empleado para las cerámicas prehistóricas del valle del Ebro (Ortega et al. 2010).

Se seleccionaron un total de 109 muestras para el análisis mineralógico mediante lámina delgada (Tab. 2). Actualmente no existe ningún procedimiento estandarizado y sistemático para la descripción petrográfica de láminas delgadas de cerámica arqueológica, a pesar de los intentos realizados (Whitbread 1989). En este trabajo la descripción petrográfica caracterizó la textura de la matriz arcillosa y las inclusiones no plásticas. En la matriz arcillosa se observó su isotropía o anisotropía, así como la morfología y distribución de los macroporos. En las inclusiones se procedió a su identificación mineralógica, se determinó sus dimensiones, morfología, índice de esfericidad y densidad relativa. El análisis petrográfico utilizó un microscopio Kiowa Biopol-2. Las micrografías se tomaron con unas cámaras Leica DFC Twain (480 R2) y Canon 450D, acopladas al trinocular del microscopio. El análisis petrográfico diferenció distintos 'grupos de manufactura' (GM) a partir de rasgos tecnológicos similares. La característica principal de estos grupos es la presencia de una mineralogía indicativa de las distintas áreas geológicas de captación, o algún rasgo tecnológico destacable como la adición de cierto tipo de desgrasante. El concepto de 'grupo de manufactura' puede ser considerado sinónimo del de 'grupo tecnopetrográfico' propuesto por otros autores (Roux y Courty 2005).

Tras la observación petrográfica de las 109 láminas delgadas, se seleccionaron 49 muestras para el análisis mineralógico mediante difracción de rayos X (DRX) y análisis geoquímico mediante espectrometría de dispersión de energías de rayos $\mathrm{X}$ (EDS). En este caso el dispositivo se acopla a un microscopio electrónico de barrido (MEB) (Tab. 2). La selección valoró que las muestras presentaran características mineralógicas distintas. El elevado coste de estas técnicas exige una selección previa del material a analizar.

Los análisis mediante DRX se llevaron a cabo con un difractómetro Bruker D8 Advance, utilizando la radiación $\mathrm{K} \alpha$ del cobre $(1,54051 \AA) \mathrm{y}$ condiciones de trabajo de $40 \mathrm{kV}$ de tensión y 30 $\mathrm{mA}$ de intensidad. Los difractogramas se registraron entre $2 \theta=5-60^{\circ}$ a temperatura ambiente, con un tiempo de integración de $8 \mathrm{~s}$ por paso en un portamuestras convencional. Los difractogramas se compararon con las bases de datos estandarizadas del Joint Committee of Powder Diffraction Standars (J.C.P.D.S.), utilizando el programa EVA suministrado por Bruker. Las muestras se molieron en mortero de ágata hasta alcanzar el estado de polvo policristalino con un diámetro

\begin{tabular}{|r|c|c|c|c|}
\hline \multicolumn{1}{|c|}{ Nivel } & Número de fragmentos & Muestras petrografía & Muestras DRX & Muestras SEM-EDS \\
\hline IV & 245 & $54(22,04 \%)$ & $23(9,39 \%)$ & $23(9,39 \%)$ \\
III & 172 & $55(31,98 \%)$ & $26(15,12 \%)$ & $26(15,12 \%)$ \\
TOTAL & 417 & $109(26,13 \%)$ & $49(11,75 \%)$ & $49(11,75 \%)$ \\
\hline
\end{tabular}

Tab. 2. Total de fragmentos cerámicos con análisis petrográficos, mineralógicos por difracción de rayos X (DRX) y geoquímicos mediante espectrometría por dispersión de energías (EDS) en microscopio electrónico de barrido (MEB) del yacimiento de Kobaederra (Cortézubi, Bizkaia), niveles III y IV. 
inferior a $30 \mu \mathrm{m}$. Para evitar el aporte de alteraciones postdeposicionales, especialmente de calcita secundaria, se desbastaron las superficies más externas de los fragmentos cerámicos.

El análisis geoquímico semicuantitativo se realizó mediante EDS sobre secciones obtenidas a partir de los fragmentos cerámicos. Tras embutirlas en una resina epoxi, se desbastaron y pulieron. Para que las secciones pulidas fueran conductoras, se recubrieron con carbono evaporado de unos 20 $\mathrm{nm}$ de espesor. Se utilizó un microscopio electrónico de barrido Jeol JSM 5800-LV, equipado con un detector de rayos X para microanálisis Oxford Instruments modelo DCL7849, realizándose los análisis con una tensión de aceleración constante de $15 \mathrm{kV}$. Los análisis mediante MEB-EDS se orientaron a obtener la composición química promedio de la matriz arcillosa, por lo que se efectuaron en zonas sin inclusiones no plásticas ni poros (Tite et al. 1982).

\section{GRUPOS DE MANUFACTURA. RESULTADOS MINERALÓGICOS Y GEOQUÍMICOS}

El análisis petrográfico indica que las materias primas empleadas en la manufactura cerámica proceden de distintas fuentes de aprovisionamiento y que los sedimentos originales se han modificado con la adición de desgrasantes, aunque los grupos de manufactura (GM) identificados en los niveles IV y III tienen cierta homogeneidad. Se han identificado 10 y 12 GM para los niveles IV y III respectivamente. Como los GM 1-7 presentan una mineralogía similar en ambos niveles se describen de forma conjunta. En cambio los grupos GM-8, GM-9, GM-10, GM-11 y GM-12 reflejan una mineralogía más heterogénea y distintiva en cada nivel. Sus principales rasgos son:

GM-1: 11 muestras (9 del nivel IV y 2 del nivel III) caracterizadas por cuarzo mono y policristalino, arenisca y sílex. El mineral arcilloso identificado es illita (Fig. 3: 1). En 2 fragmentos del nivel IV la calcita es esporádica.

GM-2: 20 muestras (16 del nivel IV y 4 del nivel III) con fragmentos de sílex. La illita es el único mineral arcilloso identificado por DRX. (Fig. 3).

GM-3: es el más numeroso con 31 muestras (16 del nivel IV y 15 del nivel III). Presenta cal- cita, aunque se observan algunas diferencias en la mineralogía del componente detrítico. La illita es el mineral arcilloso identificado en todas las muestras y la caolinita solo en algunas (Fig. 3: 2).

GM-4: 9 muestras (3 del nivel IV y 6 del nivel III). Se individualiza por la alta densidad de cuarzo mono y policristalino. Se observan también mica, plagioclasa, epidota y minerales opacos y fragmentos de roca (sílex y arenisca). El análisis mineralógico mediante DRX determina una elevada presencia de illita.

GM-5: 5 muestras (3 del nivel IV y 2 del nivel III). Se caracteriza por nódulos de arcilla y la
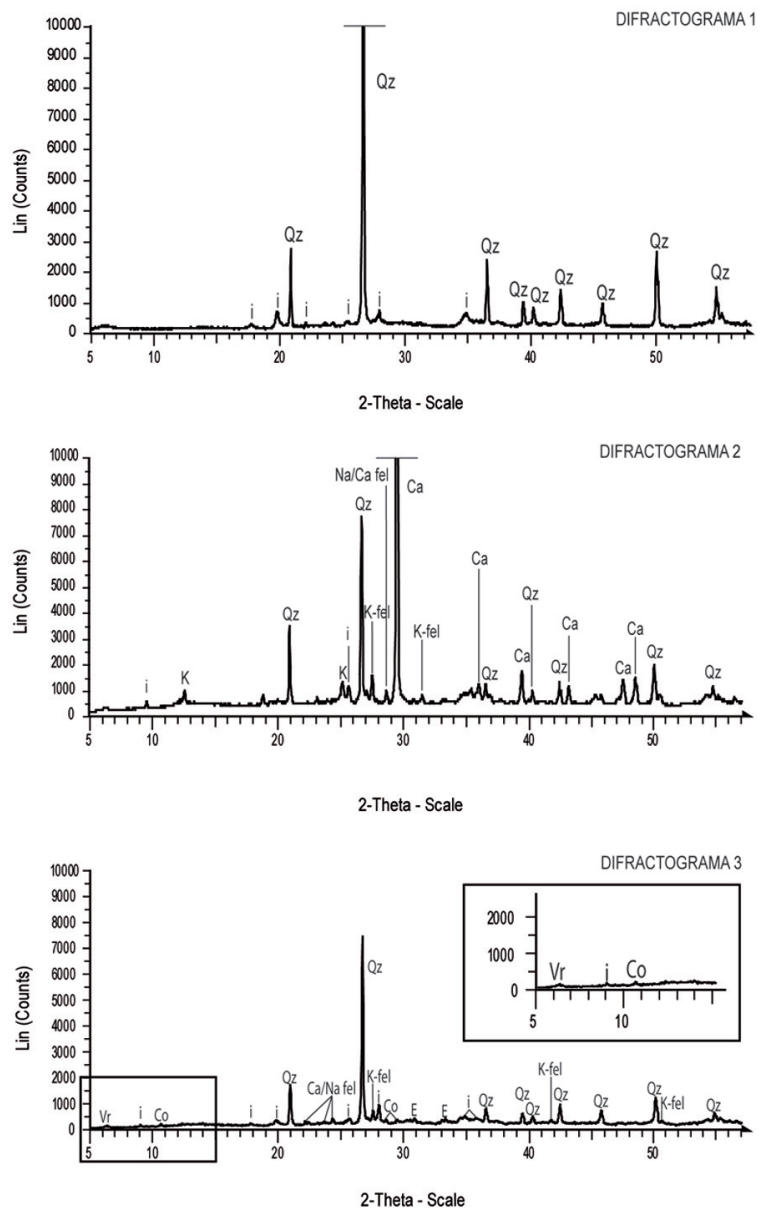

Fig. 3. Mineralogía de las cerámicas del yacimiento de Kobaederra (Cortézubi, Bizkaia). Difractogramas de las muestras: 1. GM-1, nivel IV; 2. GM-3, nivel IV; 3. GM7, nivel III. Abreviaturas: Co cordierita, E epidota, i illita, $\mathrm{K}$ caolinita, K-fel feldespato potásico, Na-Ca feldespato sódico-cálcico, Qz cuarzo, Vr vermiculita, GM Grupos de manufactura. 
identificación de illita como fase arcillosa principal. Su mineralogía, heterogénea, incluye cuarzo, epidota, e incluso, fragmentos de roca arcillosa y sílex.

GM-6: 6 muestras (2 del nivel IV y 4 del nivel III). La calcita y los minerales opacos ricos en óxido de hierro lo definen. Se ha identificado cordierita en una muestra del nivel III, lo que es coherente con la observación de cuarzos idiomorfos que la relacionan con afloramientos triásicos.

GM-7: 14 muestras ( 2 del nivel IV y 12 del nivel III). Se individualiza por los fragmentos de roca de textura ofítica. El componente detrítico comprende cuarzo, epidota y minerales de disgregación de estas rocas como piroxenos, plagioclasas y minerales opacos ricos en óxido de hierro. La illita es el mineral arcilloso más importante. En un caso se ha identificado vermiculita (Fig. 3: 3).

Los GM 8-12 reflejan una mineralogía más heterogénea y sin correlación entre los niveles IV y III. Como rasgos comunes, destacamos la chamota característica de los GM-8 del nivel IV (1 muestra) y GM-9 del nivel III (3 muestras), con un componente detrítico compuesto fundamentalmente por cuarzo monocristalino y la illita como mineral arcilloso. Los GM-9 del nivel IV (1 muestra), GM-10 (1 muestra) y 11 (1 muestra) del nivel III presentan una mineralogía poco representativa con un componente detrítico de cuarzo monocristalino, moscovita y fragmentos de roca arcillosa y caliza. La illita es el único mineral arcilloso identificado. El GM-10 (1 muestra) del nivel IV y los GM-8 (4 muestras) y 12 (1 muestra) del nivel III tienen fragmentos de arenisca. La fracción detrítica incluye cuarzo monocristalino, epidota, moscovita y minerales opacos, junto con illita como mineral arcilloso.

Según el análisis geoquímico de la matriz arcillosa se utilizan, al menos, tres posibles bancos de arcilla (Fig. 4)(3) muy similares entre sí, tal como reflejan las distancias euclídeas entre los centroi-

(3) En el análisis cluster se han considerado 49 casos (23 del nivel IV y 26 del nivel III). El análisis multivariante estudia las relaciones de las 8 variables consideradas $\left(\mathrm{SiO}_{2}, \mathrm{TiO}_{2}, \mathrm{Al}_{2} \mathrm{O}_{3}, \mathrm{FeO}\right.$, $\mathrm{MgO}, \mathrm{CaO}, \mathrm{Na}_{2} \mathrm{O}$ y $\mathrm{K}_{2} \mathrm{O}$ ) para cada muestra (nivel IV y III) sin proceder a su transformación. Se ha empleado el método de agrupación de Ward y la distancia de agrupamiento Manhattan o City Block. Posteriormente, se ha aplicado un método de optimización (k-medias) a partir del cálculo de los centroides y el número de conglomerados reconocidos en el análisis cluster para obtener las características distintivas de los grupos (Lévy y Varela 2005). des de los tres conglomerados finales calculados mediante k-medias (Tab. 3). Se diferencian en el porcentaje en peso de $\mathrm{SiO}_{2}, \mathrm{FeO}$ y $\mathrm{MgO}$, mientras que los valores de $\mathrm{CaO}$ son similares.

No se observan diferencias composicionales destacables entre los posibles bancos de arcilla utilizados en los niveles IV y III. Las composiciones geoquímicas de la matriz arcillosa no se corresponden con mineralogías concretas, lo que induce a pensar en bancos de arcilla cercanos entre sí formados a partir de un sustrato geológico similar.

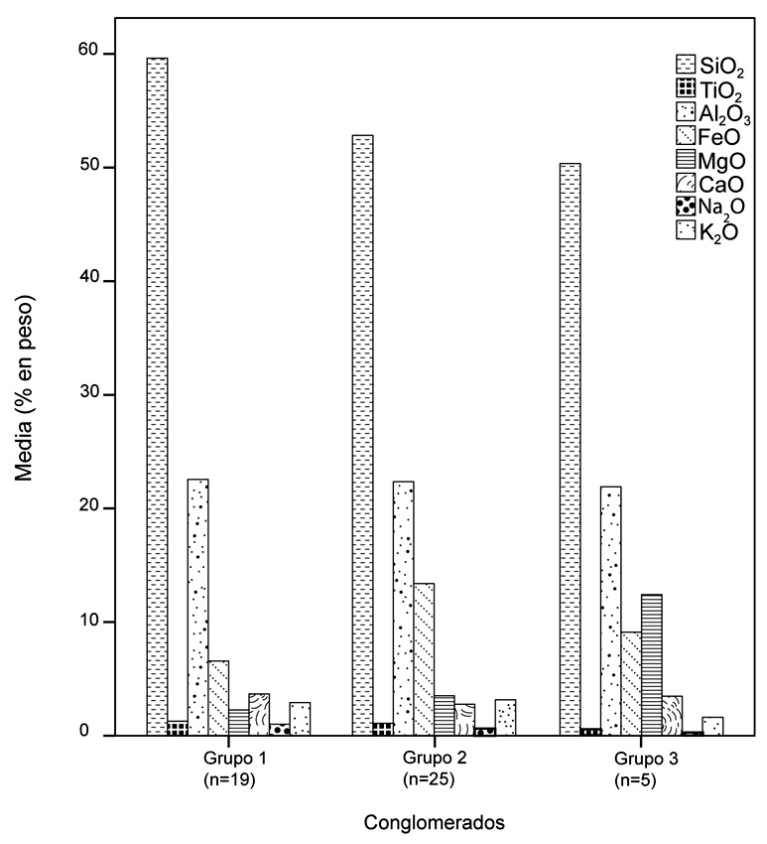

Fig. 4. Análisis geoquímico de la matriz arcillosa de la cerámica del yacimiento de Kobaederra (Cortézubi, Bizkaia). Valores promedio (\% en peso) de los óxidos presentes en los conglomerados identificados en el análisis cluster. La tabla muestra las distancias euclídeas entre los centros de los conglomerados finales establecidos mediante k-medias.

\begin{tabular}{|c|c|c|c|}
\hline Conglomerado & $\mathbf{1}$ & $\mathbf{2}$ & $\mathbf{3}$ \\
\hline 1 & & 9,635 & 17,359 \\
\hline 2 & 9,635 & & 12,878 \\
\hline 3 & 17,359 & 12,878 & \\
\hline
\end{tabular}

Tab. 3. Distancias euclídeas entre los centros de los conglomerados finales establecidos mediante k-medias. 


\section{APROVISIONAMIENTO DE MATERIAS PRIMAS}

El análisis mineralógico indica que las materias primas utilizadas para la elaboración de cerámica proceden de tres entornos geológicos distintos: de afloramientos calizos jurásicos y cretácicos, de depósitos triásicos y un tercer afloramiento detrítico relacionado con las areniscas.

La identificación de desgrasante carbonatado en algunas muestras del GM-3 de ambos niveles y la presencia de esta mineralogía en la geología de la zona sugieren el aprovisionamiento de materiales calizos. Estos afloramientos son frecuentes en el entorno de la ría de Guernica y se adscriben al Jurásico y al Cretácico. El monte Aritzgane está constituido por calizas paraarrecifales del Aptiense (Cretácico Inferior) y los depósitos jurásicos de la zona son predominantemente carbonatados (Garmendia y Robles 1991: 9). También se localizan otros materiales carbonatados como margas, dolomitas y lutitas calcáreas (IGME 1973a). El sílex del GM-2 se puede relacionar con las silicificaciones existentes en los depósitos jurásicos y cretácicos del entorno. Los afloramientos de sílex más antiguos de la cuenca vasco-cantábrica se hallan en los depósitos jurásicos (Tarriño 2006: 55) y en las formaciones carbonatadas arrecifales del complejo Urgoniano del Cretácico Inferior. En el entorno de Guernica estos afloramientos de sílex aparecen en el extremo noroeste de Munguía, en Barrica (Tarriño 2006: 63-65) y en los depósitos secundarios de Pedernales, localizados a unos $6 \mathrm{~km}$ al noroeste, cuyo uso está ampliamente documentado durante la Prehistoria.

Algunos de los grupos de manufactura evidencian la explotación de los afloramientos triásicos inmediatos al yacimiento. La mineralogía de las muestras del GM-7 es compatible con este tipo de afloramientos. Las rocas de textura ofítica identificadas son características de los depósitos triásicos (facies Keuper) localizados en el núcleo de la ría. Los suelos formados a partir de este tipo de sustrato poseen una fracción arcillosa con filosilicatos característicos como la clorita y la vermiculita (González Huecas et al. 1997: 418). La identificación de vermiculita en una muestra de este grupo (Fig. 3: 3) es un dato más que apoya la utilización de materiales triásicos. Otros GM también sugieren la utilización de este tipo de arcillas en su elaboración. Algunas muestras de los GM-3 y GM-6 contienen cuarzos idiomorfos, de morfología prismático-piramidal que, con frecuencia, exhiben inclusiones fluidas en su núcleo (Marfil 1970). Apuntan igualmente, hacia la explotación de arcillas triásicas modificadas después con la adición de otros desgrasantes.

Un tercer grupo de manufacturas (sobre todo GM-10 del nivel IV y GM-8 y 12 del nivel III) tiene fragmentos de arenisca, en general, muy abundante en el entorno del yacimiento. En la zona norte se identifica un importante depósito de cuarzoareniscas que se extiende desde Elanchove hasta el valle del $\mathrm{Ea}$ en dirección O-E y entre Nachitúa y Basecheas en dirección N-S. Esta misma formación aflora al sur del monte Aritzgane. Asimismo, al sur del yacimiento se ubican depósitos de areniscas silíceas constituidas por cuarzo, feldespato, mica blanca y materia orgánica.

Los GM que no han podido relacionarse con una litología concreta son el GM-5, caracterizado por nódulos de arcilla, y los grupos GM-8 y GM-9 con chamota. La compatibilidad entre la composición mineralógica de las cerámicas analizadas y el entorno geológico del yacimiento sugiere una producción local de las manufacturas. La utilización de materias primas procedentes de, al menos, tres entornos geológicos distintos se documenta tanto en el nivel IV como en el III, lo cual refleja una continuidad en la explotación de las materias primas destinadas a la manufactura de cerámica.

\section{MODIFICACIÓN DEL SEDIMENTO ARCILLOSO: ADICIÓN DE DESGRASANTES}

Las materias primas seleccionadas para la manufactura cerámica se pueden emplear en su estado natural o modificado. Esta modificación puede consistir en la extracción de materiales no deseados o en la adición de desgrasantes. La utilización del término 'desgrasante', como equivalente en castellano del concepto temper (Shepard 1980: 25), se entiende como la adición intencional de inclusiones no plásticas para modificar las características del sedimento original. Para distinguir una inclusión natural de una deliberada se utiliza normalmente su morfología, su tamaño, su densidad relativa o su composición mineralógica (Rice 1987: 410). 
En Kobaederra se han identificado cuatro tipos de desgrasantes: calcita, sílex, rocas de textura ofítica y chamota, lo que evidencia distintos procesos de preparación de la pasta (Fig. 5).

La calcita es el desgrasante principal determinado en algunas muestras del GM-3 de ambos niveles (Fig. 5: 1 y 2). Aparece como la mineralogía con mayor densidad relativa y se distingue del resto por su tamaño (Fig. 6) y su morfología (Fig. 5: 1 y 2). El componente detrítico de estas muestras está constituido mayoritariamente por cuarzo monocristalino de morfología redondeada
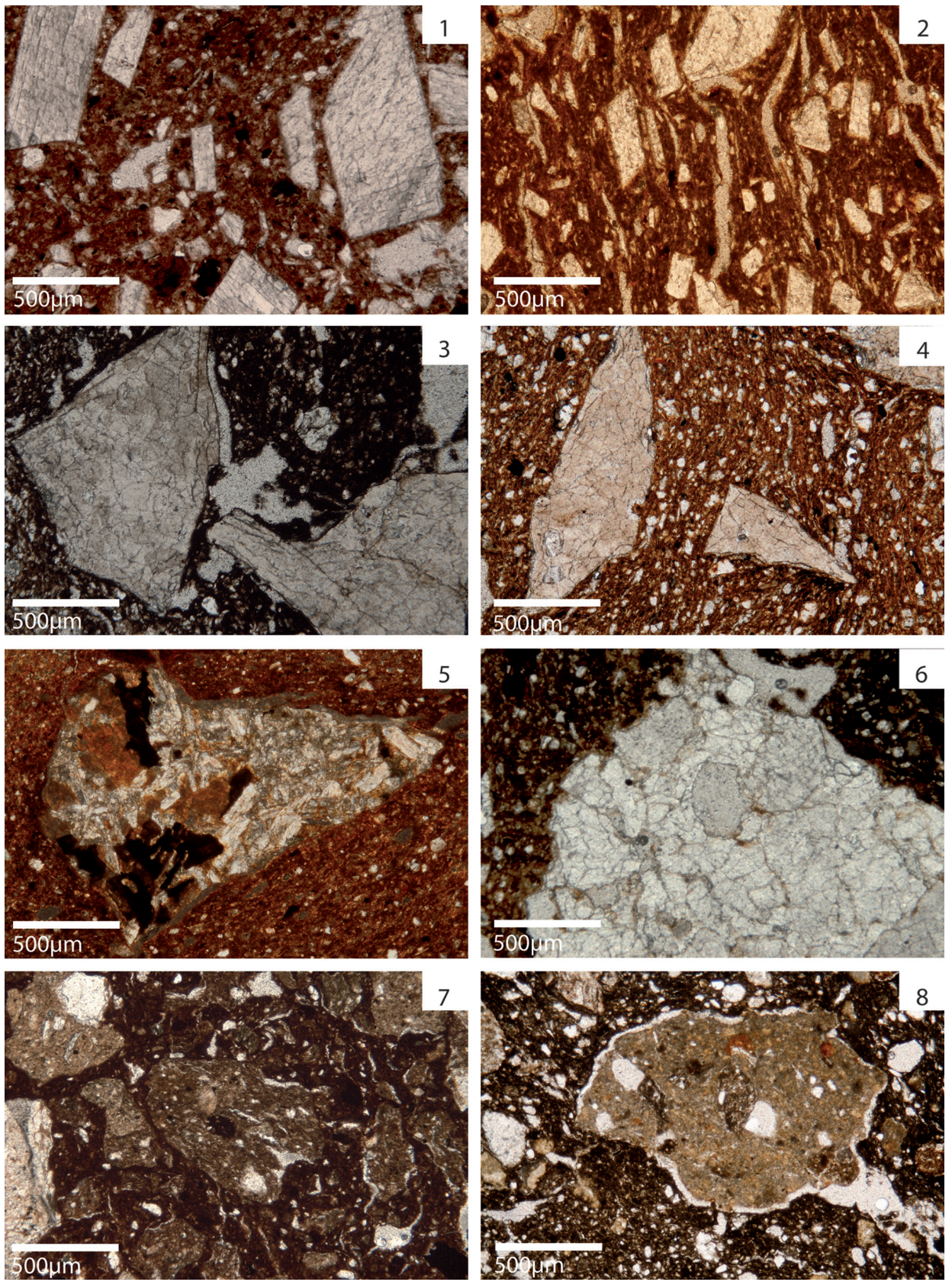

Fig. 5. Micrografías de lámina delgada (luz plana) de las inclusiones de la cerámica del yacimiento de Kobaederra (Cortézubi, Bizkaia): 1-2. calcita (GM-3); 3-4. sílex (GM-2); 5. roca de textura ofítica (GM-7, nivel III); 6. arenisca (GM-10); 7-8. chamota (GM-8, nivel IV y GM-9, nivel III). GM Grupos de manufactura. 


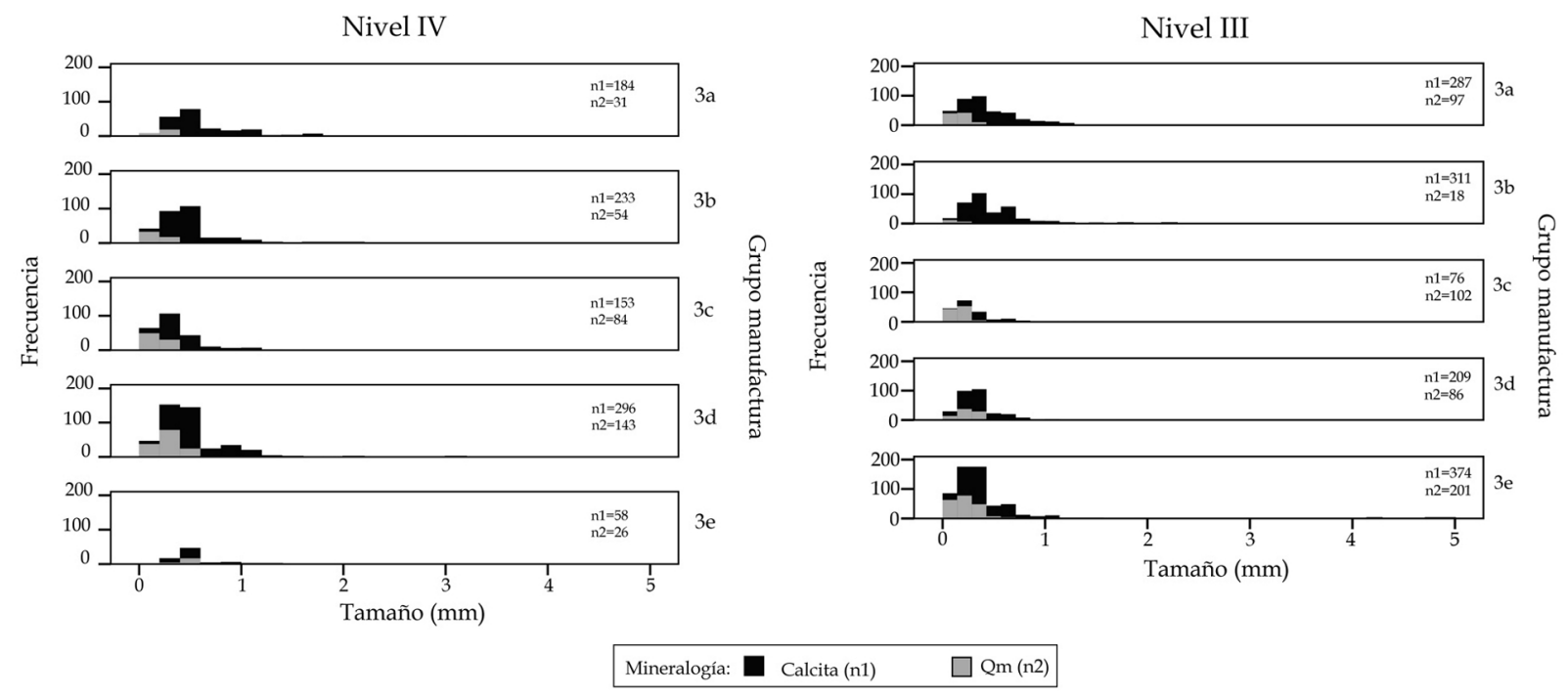

Fig. 6. Distribución del tamaño de las inclusiones de cuarzo monocristalino (Qm) y calcita en función del Grupo de manufactura (del yacimiento de Kobaederra, Cortézubi, Bizkaia).

y menores dimensiones. Su distribución de tamaño de grano es más homogénea que en la calcita. Esta última evidencia una distribución heterogénea y una morfología angulosa que puede relacionarse con una fractura intencional (Fig. 6).

El segundo de los desgrasantes es el sílex, identificado en algunas muestras del GM-2 de ambos niveles. Sus inclusiones son de morfología angulosa, gran tamaño y una especial recurrencia, aunque su densidad relativa es menor que la de la calcita (Fig. 5: 3 y 4).

Los fragmentos de roca de textura ofítica se han considerado producto de una adición intencionada en las muestras clasificadas en el GM-7 del nivel III. Tienen mayor tamaño que el componente detrítico y una morfología marcadamente angulosa, aunque su densidad relativa es baja (Fig. 5: 5). Los fragmentos de arenisca identificados presentan una morfología claramente angulosa y un tamaño mayor que el del componente detrítico, pero no se ha podido determinar, con los criterios descritos anteriormente su intencionalidad (Fig. 5: 6).

La adición de chamota se detecta en algunas muestras de los GM-8 y GM-9 del nivel III y IV respectivamente (Fig. 5: 7 y 8). Puede considerarse un desgrasante artificial, ya que se trata de fragmentos de cerámica machacada (Rice 1987: 75). Solo se clasificaron como tal las inclusiones con una clara diferenciación perimetral con respecto a la matriz arcillosa y características distintivas respecto a esta, como birrefringencia o coloración.

\section{CRONOLOGÍA Y SECUENCIAS DE MANUFACTURA}

Las dataciones absolutas disponibles para los niveles IV y III de la secuencia de Kobaederra indican que su formación se produce entre la primera y la segunda mitad del V milenio cal AC. A pesar de su imprecisión (Tab. 1), el solapamiento entre las dataciones de ambos niveles apunta a que fue poco dilatada en el tiempo. Aunque se ha identificado un elevado número de $\mathrm{GM}$, la variabilidad tecnológica observada en los conjuntos cerámicos procedentes de los niveles IV y III no es especialmente significativa, lo cual indica que podrían ser casi coetáneos. No obstante, se pueden señalar algunos aspectos importantes respecto a su secuencia de manufactura.

El aprovisionamiento de materias primas es bastante similar en los dos niveles, ya que sus materiales cerámicos se han manufacturado con materias primas locales del entorno geológico inmediato al yacimiento. Se han identificado, además, los mismos tipos de desgrasantes, salvo 
la adición de rocas de textura ofítica propia del nivel III (12 muestras).

La calcita es el desgrasante con mayor representación en ambos niveles ( 25 muestras del GM3), mientras el sílex es mayoritario en el nivel IV (11 muestras) y desciende de forma importante en el nivel III (4 muestras). El empleo de la chamota es ocasional (3 muestras) en ambos niveles. Se observa, por tanto, que el sílex es más abundante en el nivel inferior de la secuencia, mientras que las rocas de textura ofítica se utilizan de forma sistemática sólo en el nivel superior.

La utilización de estos desgrasantes, especialmente la calcita, se documenta durante el Neolítico en todo el ámbito de la Península Ibérica. La calcita aparece en la región cantábrica, con cronologías similares, en el yacimiento de Los Gitanos (Cubas y Ontañón 2009). Otra zona de dispersión es el valle del Ebro, en yacimientos como Mendandia (Ortega et al. 2010) y Cueva Lóbrega (Gallart y Mata 2004) entre el VII y el VI milenio cal AC, hasta contextos en la desembocadura a partir del VI milenio cal AC (Martín et al. 2010). A estos enclaves debe añadirse Chaves en el Prepirineo de Huesca (Gallart y López 1988). Una tercera zona de dispersión es la levantina, donde aparece desde los inicios del Neolítico en la Cova de l'Or (Gallart 1980; McClure et al. 2006; McClure y Molina 2008) y Mas d'Is (McClure et al. 2006). En el sur de la Península Ibérica, se identifica en varios yacimientos de Andalucía (Navarrete et al. 1991; Lazarich et al. 2002).

El uso del resto de desgrasantes está menos extendido en el territorio peninsular. El sílex se documenta en La Carigüela (Navarrete et al. 1991) en cronologías neolíticas. Las rocas de textura ofítica aparecen en la Cueva de los Murciélagos de Zuheros (Barrios et al. 1999) y en Papa Uvas (Barrios et al. 2005) en cronologías ligeramente más recientes. Asimismo, se identifica en la Edad del Hierro en los yacimientos de la Cuenca de Pamplona (Olaetxea 2000). La chamota aparece en un elevado número de yacimientos peninsulares del valle alto-medio del Ebro (Ortega et al. 2010), la zona catalana (Clop 2007), Valencia (McClure et al. 2006), Madrid (Díazdel-Río et al. 2011) y Andalucía (Navarrete et al. 1991). Su uso en la región cantábrica parece que se mantiene hasta la Edad del Hierro (Olaetxea 2000).

\section{CONSIDERACIONES FINALES}

El estudio tecnológico evidencia importantes similitudes entre los conjuntos cerámicos de los niveles IV y III. Esta homogeneidad corrobora la hipótesis apuntada a partir de la industria lítica, el espectro arqueobotánico, malacológico y faunístico (Zapata et al. 1997; Zapata 2002; Gutiérrez Zugasti 2009, Altuna y Mariezkurrena 2009) sobre la similitud de las ocupaciones antiguas del yacimiento (Zapata et al. 1997: 57).

Destacamos el uso de instrumentos de sílex para el trabajo de la cerámica en los niveles IV y III (Ibáñez 2001: 232). Se trata de dos láminas de sílex con huellas de uso relacionadas con el raspado de un material mineral abrasivo, utilizando agua como lubricante. El hallazgo de estos útiles es poco frecuente en contextos arqueológicos, aunque se conoce en yacimientos como L'Eglise (Gassin 1996) y la cueva de El Toro (Rodríguez et al. 1996), y debe ponerse en relación con los procesos de modelado previos a la cocción del recipiente. Su presencia y la disponibilidad de materias primas en el entorno sugieren una producción local. Se observa una continuidad en la explotación de los posibles bancos de arcilla y en la utilización de desgrasantes. La comparación con el resto del conjunto arqueológico permite apuntar un carácter habitacional del yacimiento en estos momentos ya que se han identificado actividades realizadas en la propia cavidad. En este contexto, la aparición de la cerámica debe interpretarse como un elemento más de las actividades cotidianas de subsistencia de sus habitantes.

\section{AGRADECIMIENTOS}

Este trabajo se enmarca en el proyecto "La implantación de las especies domésticas en la Europa atlántica: cronología e impacto en la dieta humana" (DOMATLANTICA, HAR200806477-C03-01/HIST), financiado por el VI Plan Nacional de Investigación Científica, Desarrollo e Innovación Tecnológica 2008-2011 del Ministerio de Ciencia e Innovación. Miriam Cubas disfrutó de una beca predoctoral de la Universidad de Cantabria y realizó durante una estancia de dos meses en el Centro de Ciencias Humanas y Sociales-CSIC el trabajo de observación petrográfica, a principios de 2009, bajo la dirección del 
Dr. Manuel García-Heras. Los autores agradecen al Dr. Eneko Iriarte su colaboración en esta investigación.

El trabajo de la Dra. Lydia Zapata forma parte del Grupo de Investigación de la Universidad del País Vasco UPV/EHU IT-288-07 financiado por el Gobierno Vasco y de los Proyectos HAR200803976/HIST del MICIIN y AGRIWESTMED (Origins and spread of agriculture in the western Mediterranean region) del ERC (ERC-AdG230561).

\section{BIBLIOGRAFÍA}

Alday, A. 2003: “Cerámica neolítica en la región vasco-riojana: base documental y cronológica". Trabajos de Prehistoria 60 (1): 19-50.

Altuna, J. y Mariezkurrena, K. 2009: “Tipos de cabañas ganaderas durante el Neolítico en el País Vasco y zonas próximas". Archaeofauna 18: 137-157.

Apellániz, J. M. y Altuna, J. 1975: "Memoria de la III campaña de excavaciones arqueológicas en la cueva de Arenaza I (San Pedro de Galdames, Bizkaia)". Noticiario Arqueológico Hispánico. Prehistoria 4: 183-197.

Arias, P. 1991: De cazadores a campesinos. La transición al neolítico en la región cantábrica. Universidad de Cantabria. Santander.

Arias, P. 2007: "Neighbours but diverse: social change in north-west Iberia during the transition from the Mesolithic to the Neolithic (5500-4000 cal BC)". En A. Whittle y V. Cummings (eds.): Going over: the Mesolithic-Neolithic transition in North-West Europe. Proceedings of the British Academy 144, Oxford University Press. Oxford: 53-72.

Arias, P. y Altuna, J. 1999: "Nuevas dataciones absolutas para el Neolítico de la cueva de Arenaza (Bizkaia)". Munibe (Antropologia-Arkeologia) 51: 161-171.

Arias, P.; Altuna, J.; Armendáriz, A.; González Urquijo, J. E.; Ibáñez Estévez, J. J.; Ontañón, R. y Zapata, L. 1999: "Nuevas aportaciones al conocimiento de las primeras sociedades productoras en la región cantábrica”. En J. Bernabéu y T. Orozco (eds.): Actas del II Congrés del Neolític a la Península Ibérica (Valencia, 1999). Saguntum. Papeles del Laboratorio de Arqueología de Valencia, Extra 2, Valencia: 549-557.

Arias, P.; Altuna, J.; Armendáriz, A.; González Urquijo, J. E.; Ibáñez Estévez, J. J.; Ontañón, R. y Zapata, L. 2000: "La transición al neolítico en la región cantábrica. Estado de la cuestión". En P. Arias, P. Bueno, D. Cruz, J. X. Enríquez, J. Oliveira y M. J. Sanches (eds.): Neolitizaçao e megalitismo da Península Ibérica. Actas do 3. ${ }^{\circ}$ Congresso de Arqueologia Peninsular (Vila Real, 1999): 115-133. Porto.

Barrios, J.; Gavilán, B.; Martínez, M. J. y Montealegre, L. 1999: “Caracterización de cerámicas neolíticas procedentes de la Cueva de los Murciélagos, Córdoba". En J. Capel (ed.): Arqueometría y arqueología. Universidad de Granada. Granada: 49-55.

Barrios, J.; Montealegre, L.; Martín de la Cruz, J. C.; Lucena Martín, A. M. y Lucena Martín, J. M. 2005: "Repertorios materiales de Papa Uvas (Aljadraque, Huelva): caracterización mineralógica y estructural". En P. Arias, R. Ontañón y C. García-Moncó (eds.): III Congreso del Neolítico en la Península Ibérica (Santander, 2003): 369-372. Santander.

Bohigas, R. y Muñoz, E. 2002: "Excavaciones arqueológicas de urgencia en el covacho de Arenillas (Islares, Castro Urdiales) 1992”. En R. Ontañón (ed.): Actuaciones arqueológicas en Cantabria 19871999. Gobierno de Cantabria. Santander: 45-47.

Clop, X. 2007: Materia prima, cerámica y sociedad. La gestión de los recursos minerales para manufacturas cerámicas del 3100 al 1500 ANE en el noreste de la Península Ibérica. British Archaeological Reports, International Series 1660, Archaeopress. Oxford.

Cowgill, G. L. 1964: "The selection of samples from large sherd collections". American Antiquity 29: 467-473.

Cubas, M. 2008: "La producción cerámica en la primera mitad del V milenio cal BC: el caso de la Región cantábrica”. En M. S. Hernández, J. A. Soler y J. A. López (eds.): IV Congreso del Neolítico Peninsular (Alicante, 2006): 282-289. Alicante.

Cubas, M. y Ontañón, R. 2009: "The material evidence of the 'production sequence'. The case of the pottery ensemble of Los Gitanos cave (Castro Urdiales, Cantabria, Spain)". Journal of Iberian Archaeology 12: 7-22.

Díaz-del-Río, P.; Consuegra, S.; Domínguez, R.; Martín-Bañón, A.; Vírseda, L.; Agua, F.; Villegas, M. A. y García-Heras, M. 2011: "Identificación de una tradición tecnológica cerámica con desgrasante óseo en el Neolítico peninsular. Estudio arqueométrico de materiales cerámicos de Madrid (5300-3400 cal AC)". Trabajos de Prehistoria 68 (1): 99-122.

Ente Vasco de la Energía (EVE) 2003: Mapa geológico del País Vasco E: 1:25.000. Edición digital. Bilbao.

Gallart, M. D. 1980: "La tecnología cerámica”. En B. Martí, V. Pascual, M. D. Gallart, P. López, M. Pérez, J. D. Acuña, y F. Robles (eds.): Cova de l'Or. Serie de Trabajos Varios 25, Diputación de Valencia. Valencia: 165-173.

Gallart, M. D. y López, F. 1988: “Análisis mineralógico de las cerámicas neolíticas de la cueva de Chaves (Casbas, Huesca)". Bolskam 5: 5-26. 
Gallart, M. D. y Mata, M. P. 2004: “Análisis mineralógico y textural del grupo cerámico de Cueva Lóbrega”. En I. Barrios Gil (ed.): El yacimiento de cueva Lóbrega (Torrecilla en Cameros, La Rioja). Una visión acerca del Neolítico y la Edad del Bronce en el área occidental del Sistema Ibérico. Instituto de Estudios Riojanos. Logroño: 159-181.

Garmendia, J. M. y Robles, S. 1991: "Litoestratigrafía del Jurásico en Gipuzkoa y NW de Navarra". Geogaceta 10: 6-10.

Gassin, B. 1996: Évolution socio-économique dans le Chaséen de la grotte de l'Églisse supérieur (Var). CNRS. París.

González Huecas, C.; Valverde, I. y López, A. 1997: "Alteración mineralógica de suelos desarrollados sobre ofitas". Boletín de la Sociedad Española de la Ciencia del Suelo 3-2: 413-419.

González Morales, M. R. y Straus, L. G. 2000: “El depósito arqueológico de la Prehistoria Reciente de la cueva del Mirón (Ramales, Cantabria): bases estratigráficas para una secuencia regional". En P. Bueno, J. L. Cardoso, M. Díaz-Andreu, V. Hurtado, S. Oliveira y V. Oliveira (ed.): Pré-História recente de Península Ibérica Actas do 3. ${ }^{\circ}$ Congresso de Arqueologia Peninsular (Vila Real, 1999): 49-63. Porto.

Gutiérrez Zugasti, F. I. 2009: "Explotación de recursos costeros durante el Neolítico en el área de la Reserva de Urdaibai: los moluscos de la cueva de Kobaederra (Kortézubi, Bizkaia)". Munibe (Antropologia-Arkeologia) 60: 175-186.

Ibáñez Estévez, J. J. 2001: "La función de los útiles retocados del yacimiento de Kobaederra (Oma, Bizkaia). Campañas de 1995, 96 y 97'. Isturiz 11: 225-244.

Instituto Geológico y Minero de España (IGME). 1973a: Bermeo: mapa geológico de España. Escala 1:50.000 Hoja 38. Servicio de Publicaciones. Ministerio de Industria y Energía, D. L. Madrid.

Instituto Geológico y Minero de España (IGME). 1973b: Durango: mapa geológico de España. Escala 1:50.000 Hoja 62. Servicio de Publicaciones. Ministerio de Industria y Energía, D. L. Madrid.

Iriarte, M. J.; Mujika, J. A. y Tarriño, A. 2005: "Herriko Barra (Zarautz-Gipuzcoa): caractérisation industrielle et économique des premiers groupes de producteurs sur le littoral basque". Unité et diversité des processus de neolithisation sur la façade atlantique de l'Europe. Mémoire XXXVI, Société Prehistorique Française. París: 127-136.

Lazarich, M. 2002: “Análisis tecnológico, morfológico y funcional de los productos cerámicos de 'El Retamar'”. En J. Ramos y M. Lazarich (eds.): El asentamiento de 'El Retamar' (Puerto Real, Cádiz). Contribución al estudio de la formación social tribal y a los inicios de la economía de producción de la Bahía de Cádiz. Universidad de Cádiz. Cádiz: 363-376.
Lévy, J. P. y Varela, J. 2005: Análisis multivariable para las ciencias sociales. Pearson Educación. Madrid.

Marfil, R. 1970: "Estudio petrogenético del Keuper en el sector meridional de la Cordillera Ibérica". Estudios Geológicos 26: 113-161.

Martín, A.; Edo, M.; Tarrús, J. y Clop, X. 2010: “Le Néolithique ancien de Catalogne ( $\mathrm{VI}^{\mathrm{e}}$-première moitié du $V^{e}$ millénaire av J. C.). Les séquences chronoculturelles". En C. Manen, F. Convertini, D. Binder e I. Sénépart (eds.): Premières sociétés paysannes de Méditerranée occidentale. Structures des productions céramiques. Mémoire LI, Société Préhistorique Française. París: 197-214.

McClure, S. D.; Bernabeu, J.; García, O.; Aura, E.; Molina, L.; Descantes, C.; Speakman, R. J. y Glascock, M. D. 2006: "Testing technological practices: neutron activation analysis of Neolithic ceramics from Valencia, Spain". Journal of Archaeological Science 33 (5): 671-680.

McClure, S. D. y Molina, L. 2008: "Neolithic ceramic technology and Cardial ware in the Alcoi basin, Valencia". En M. S. Hernández, J. A. Soler y J. A. López (eds.): IV Congreso del Neolítico Peninsular (Alicante, 2006): 298-304. Alicante.

Navarrete, M. S.; Capel, J.; Linares, J.; Huertas, F. y Reyes, E. 1991: Cerámicas neolíticas de la provincia de Granada. Materias primas y técnicas de manufacturación. Universidad de Granada. Granada.

Neff, H. 1993: "Theory, sampling and analytical techniques in the archaeological study of prehistoric ceramics". American Antiquity 58: 23-44.

Olaetxea, C. 2000: La tecnología cerámica en la protohistoria vasca. Munibe supl. 2, Sociedad de Ciencias Aranzadi. San Sebastián.

Ontañón, R. 2005: "La secuencia de la Cueva de Los Gitanos (Castro Urdiales, Cantabria) y el Neolítico cantábrico". En P. Arias, R. Ontañón y C. García-Moncó (eds.): III Congreso del Neolítico en la Península Ibérica (Santander, 2003): 1035-1043. Santander.

Ortega, L. A.; Zuluaga, M. C.; Alonso-Olazabal, A.; Murelaga, X. y Alday, A. 2010: "Petrographic and geochemical evidence for long-standing supply of raw materials in Neolithic pottery (Mendandia site, Spain)". Archaeometry 52 (6): 987-1001.

Peña-Chocarro, L.; Zapata, L.; Iriarte, M. J.; González Morales, M. R. y Straus, L. G. 2005: "The oldest agriculture in northern Atlantic Spain: new evidence from El Mirón Cave (Ramales de la Victoria, Cantabria)". Journal of Archaeological Science 32 (4): 579-587.

Ramsey, C. B. 2001: "Development of the radiocarbon calibration program". Radiocarbon 43 (2A): 355-363.

Ramsey, C. B. 2009: "Bayesian analysis of radiocarbon dates". Radiocarbon 51 (1): 337-360. 
Reimer, P. J.; Baillie, M. G. L.; Bard, E.; Bayliss, A.; Beck, C. W.; Bronk Ramsey, C.; Buck, C. E.; Burr, G. S.; Edwards, R. L.; Friedrich, M.; Grootes, P. M.; Guilderson, T. P.; Hajdas, I.; Heaton, T. J.; Hogg, A. G.; Hughen, K. A.; Kaiser, K. F.; Kromer, B.; McCormar, F. G.; Manning, S. W.; Reimer, R. W.; Richards, D. A.; Southon, J. R.; Talamo, S.; Turney, C. S. M.; Van der Plicht, J. y Weyhenmeyer, C. E. 2009: "IntCal09 and Marine09 radiocarbon age calibration curves, $0-50,000$ years cal BP". $R a$ diocarbon 51 (4): 1111-1150.

Rice, P. M. 1987: Pottery analysis. A sourcebook. University of Chicago Press. Chicago.

Rodríguez Rodríguez, A. C.; Martín, D.; Massieu, C. y González Quintero, P. 1996: "Las actividades tecnoeconómicas en Cueva del Toro (Antequera, Málaga) a través del análisis funcional". I Congrés del Neolític a la Península Ibérica (Gavà, 1993): 161-167. Gavà.

Roux, V. y Courty, M. A. 2005: "Identifying social entities at a macro-regional level: Chalcolithic ceramics of South Levant as a case of study". En A. Livingstone, D. Bosquet y R. Martineau (eds.): Pottery manufacturing processes: reconstitution and interpretation. British Archaeological Reports, International Series 1349, Archaeopress. Oxford.

Shepard, A. O. 1980: Ceramics for the archaeologist. Carnegie Institution of Washington. Washington, D. C.
Tarriño, A. 2006: El sílex en la cuenca vasco-cantábrica y el Pirineo navarro. Ministerio de Cultura. Madrid.

Tite, M. S.; Freestone, I. C.; Meeks, N. D. y Bimson, M. 1982: "The use of Scanning Electron Microscopy in the technological examination of ancient pottery". En J. Olin y A. Franklin (eds.): Archaeological ceramics. Smithsonian Institution Press. Washington D. C.: 109-120.

Whitbread, I. K. 1989: "A proposal for the systematic description in thin sections towards the study of ancient ceramic technology". En Y. Maniatis (ed.): Archaeometry. Elsevier Science Publishers B. V. Amsterdam: 127-138.

Zapata, L. 2002: Origen de la agricultura en el Pais Vasco y transformaciones en el paisaje: análisis de restos vegetales arqueológicos. Kobie anejo 4, Diputación Foral de Bizkaia. Bilbao.

Zapata, L.; González Urquijo, J. E. e Ibáñez Estévez, J. J. 1997: "El yacimiento de la cueva de Kobaederra Oma, Kortezubi, Bizkaia. Resultados preliminares de las campañas de excavación 1995-97”. Munibe (Antropologia-Arkeologia) 49: 51-63.

Zapata, L.; González Urquijo, J. E.; Ibáñez Estévez, J. J.; Altuna, J.; Mariezkurrena, K. y Rúa, C. de la 2000: "Condiciones ambientales y aprovechamiento de recursos en el Neolítico. El yacimiento arqueológico de Kobaederra Oma, Kortezubi”. Investigación aplicada a la reserva de Urdaibai. UNESCO Etxea-Gobierno Vasco. Bilbao: 221-228. 